\title{
La prévention: promotion de la santé ou thérapie individuelle?
}

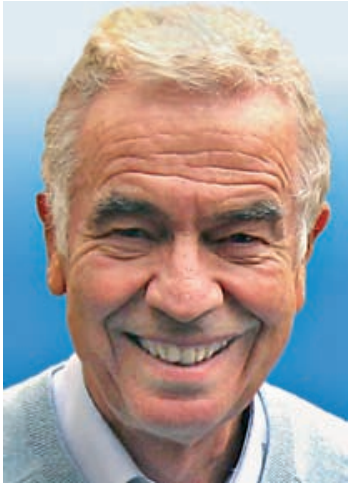

Hans Stalder
* Le Prof. Hans Stalder, médecin spécialiste en médicine interne FMH, membre de la rédaction, était médecin-chef de la Policlinique de médecine et du Département de médecine communautaire des Hôpitaux universitaires de Genève.
Le Conseil fédéral vient de proposer un avantprojet de loi destiné à renforcer la prévention. C'est certainement louable, car la Suisse, comparée à d'autres pays, n'est pas très avancée dans ce domaine. Chez nous, les dépenses pour la prévention s'élèveraient «seulement» à 1,13 milliards de francs, soit 2,2\% du budget de la santé, tandis que la moyenne dans les pays de l'OCDE se situe à 2,7\% et dans les Pays-Bas même à $5,5 \%$. Ce chiffre induit cependant en erreur.

On oublie que depuis longtemps en médecine clinique on fait aussi de la prévention: plus d'un tiers des consultations de pédiatrie et de gynécologie/obstétrique et une bonne partie de l'activité du généraliste sont dévolues à des actions préventives. Quand il s'agit de conseils (par exemple en pédiatrie) ou de dépistage (en gynécologie), on le fait ouvertement et c'est une excellente chose. Ça devient plus problématique quand on cache la prévention sous le manteau $\mathrm{du}$ «traitement» et quand on appelle «maladie» ce qui ne sont en réalité que des facteurs de risque, tels que tension artérielle, glycémie et cholestérol élevés. On oublie qu'il s'agit là uniquement de mesures cliniques ou de laboratoire dont les valeurs «normales» sont par ailleurs continuellement abaissées par des soi-disant experts, dont on peut douter de leur indépendance et qui ont réussi à rendre «malade» pratiquement toute la population «normale» âgée de plus de 50 ans, car qui ne dépasserait pas au moins une des trois valeurs? On peut alors se demander ce que veut dire «normal» (mais cela sera peut-être le sujet d'un autre «et encore ...»). Ainsi, on «traite» ces facteurs de risque et encore bien d'autres, car ce sont dorénavant des «maladies», même s'il s'agit en fait de la prévention de pathologies ultérieures. Est-ce alors étonnant que les patients, qui ne se sentent pas et, en réalité, ne sont pas malades, ne suivent pas ce «traitement»?

Il y a d'autres raisons qui font que ces «traitements» ne sont pas très efficients. L'effet annoncé est souvent trompeur car indiqué en diminution mirobolante de risques relatifs, à la place de risques absolus qui se comptent en quelques pourcentages. C'est pourquoi des dizaines voire des centaines de patients doivent être traités pendant plusieurs années pour voir l'effet de ce «traitement» qui coûte par ailleurs très cher. (Notons qu'il y a ici une différence importante comparée aux vaccinations qui, même en ne diminuant que légèrement les risques individuels d'attraper la maladie, servent à protéger la population entière.) De plus, «traiter» les facteurs de risque n'est pas identique à diminuer l'incidence de maladies ultérieures: on se souvient des mésaventures avec la cérivastine, la rosiglitazone, la vitamine $\mathrm{E}$ ou encore les estrogènes ... Enfin, par «traitement» médecins et patients sousentendent en général prescription de médicaments, par ailleurs proposés par les mêmes experts «indépendants», qui abaissent les seuils de normalité, même si on sait que des changements d'habitudes telles que la cigarette ou l'inactivité physique sont au moins aussi efficaces si ce n'est plus. Mais justement: les médicaments pour ces «maladies» sont payés par les caisses tandis que mes chaussures de gymnastique ne le sont pas ...

Il y a donc prévention et prévention. Pour autant qu'il faille être critique pour la poursuite exagérée de facteurs de risques individuels en rendant les patients malades quand ils ne le sont pas, il faut soutenir l'initiative de promouvoir la santé au niveau de la population. Ici, les facteurs de risque de morbidité et de mortalité les plus importants ne sont cependant pas d'ordre médical, mais socio-économique. Des actions sociales, environnementales, régulatrices (par exemple sur l'alimentation) et d'information doivent donc être entreprises. Il n'y a pas de doute, la promotion de la santé pour les populations à risque - on ferait mieux de laisser ici tomber le terme "prévention» - n'est pas la même chose que la prévention individuelle au cabinet. Pour être efficace, la promotion de la santé impose une action étatique qui, étant quelquefois contraignante, est continuellement combattue par ceux qui combinent (ou confondent?) la défense de la liberté individuelle avec leurs intérêts économiques.

Hans Stalder* 